\title{
Contributors to the 2017 IMIA Yearbook of Medical Informatics
}

Editors:

John H. Holmes

University of Pennsylvanio

Perelman School of Medicine

Phildadphia, Pennsylvania, USA

jhholmes@mail.med..vpenn.edu

Brigitte Séroussi

UPMC Univ Paris $06 \&$ LIMIIS UMRS 1142

Tenon Hospital, AP-HP

Paris, France

brigitte.seroussi@aphp.fr

Lina F. Soudmia

Normandie Universités, Univ. Roven

LITIS, Information Processing in Biology \& Health

Roven, France

Lina.Soudmia@chu-roven.fr

Editorial Assistant:

Martina Hutter

Heidelberg University Hospital- ZIM

Heidelberg, Germany

yearbook@imia-services.org

\section{Best Papers Editor:}

Adrien Ugon

Université Pierre et Marie Curie

adrien.ugon@lipb.fr

\section{Advisory Board:}

Reinhold Houx

Peter L. Reichertz Institute for Medical Informatics

University of Braunschweig - Institute of Technology

and

Hannover Medical School

Braunschweig, Germany

reinhold.haux@plri.de

Victor Maojo

Universidad Politécnica de Madrid

Departamento de Inteligencia Artificial

Facultad de Informatica

Madrid, Spain

vmaojo@ffi.upm.es

George Mihalas

University of Medicine and Pharmacy

Dept. of Medical Informatics

Timisoara, Romania

mihalas@EFMl.info

\section{Section Editors:}

Eto S. Berner

Department of Health Services Administration

University of Alabama at Birmingham

Birmingham, AL, USA

eberner@uab.edu

Meryl Bloomrosen

Premier healthcare alliance

Washington, $D C$

meryl_bloomrosen@premierinc.com
Jacques Bouaud

AP-HP \& LIMICS UMRS 1142

Paris, France

jacques.bouaud@aphp.fr

Jean Charlet

AP-HP \& LIMICS UMRS 1142

Paris, France

jean.charlet@upmc.fr

Rémy Choquet

BNDMR, AP-HP \& LIMICS, UMRS 1142

Paris, France

remy.choquet@aphp.fr

Christel Daniel

AP-HP WIND-DSI \& LIMICS UMRS 1142

Paris, France

christel.daniel@aphp.fr

Hélène Dauchel

Normandie Univ., UNIROUEN

LITIS, Team Information Processing in Biology \&

Health (TIBS)

Roven, France

helene.dauchel@univ-rouen.fr

Ferdinand Dhombres

INSERM, UMR_S 1142, LIMICS

Paris, France

ferdinand.dhombres@inserm.fr

Luis Fernandez-Luque

Qatar Computing Research Institute

Hamad Bin Khalifa University

Doha, Qatar

lluque@qf.org.qa

Grégoire Ficheur

Department of Medical Informatics, EA 2694

Lille University Hospital, Lille, France

gregoire.ficheur@univ-lille2.fr

Thomas Ganslandt

Medizinisches IK-Zentrum

Universitätsklinikum Erlangen

Erlangen, Germany

Thomas.Ganslandt@uk-erlangen.de

Werner Hackl

Institute of Biomedical Informatics

UMIT - University for Health Sciences, Medical

Informatics and Technology

Hall in Tirol, Austria

werner.hack@@umit.at

William Hsu

Department of Radiological Sciences

University of California Los Angeles

Los Angeles, CA, USA

whsu@mednet.ucla.edu

Charles E. Kahn, Jr.

Department of Radiology

University of Pennsylvania

Philadelphia, PA, USA

charles.kahn@uphs.upenn.edu
Vassilis Koutkias

Institute of Applied Biosciences

Centre for Research \& Technology Hellos

Thermi, Thessaloniki, Greece

vkoutkias@certh.gr

Thierry Lecrog

Normandie Univ., UNIROUEN

LITIS, Team Information Processing in Biology \&

Health (TIBS)

Rouen, France

thierry.lecrog@univ-roven.fr

Aurélie Névéol

LIMSI, CNRS, Université Paris-Saclay

Orsay, France

neveol@limsi.fr

Soojin Park

Department of Neurology

Columbia University College of Physicians and

Surgeons

New York, NY, USA

sp3291@cumc.columbia.edu

Sylvia Pelayo

INSERM CIC-IT 1403 Evalab

Université Lille 2, CHU de Lille

Lille, France

sylvia.pelayo@univ-lille2.fr

Raquel Santos

Hospital Da Luz - Learning Health

Lisboa, Portugal

raquel.santos@luzsaude.pt

Daniel R. Schlegel

Department of Computer Science

SUNY Oswego

Oswego NY, USA

daniel.schlegel@oswego.edu

Pascal Staccini

INSERM UMR 912

Université Nice-Sophia Antipolis

Nice, France

pascal.staccini@unice.fr

Rodolphe Thiébaut

Univ. Bordeaux, Inserm

UMR 1219

Bordeaux, France

rodolphe.thiebout@u-bordeaux.fr

Frantz Thiessard

Univ. Bordeaux, Inserm,

Bordeaux Population Health Research Center

UMR 1219

Bordeaux, France

frantz.thiessard@isped.u-bordeaux2.fr

Pierre Zweigenboum

LIMSI, CNRS, Université Paris-Saclay

Orsay, France

pz@limsi.fr
Regional Editors:

Karen Greenwood

AMIA

Bethesda, MD, USA

karen@amia.org

Jeremy Theal

COACH: Canada's Health Informatics Association

Toronto, ON, Canada

Jeremy.Theal@nygh.on.ca

Alexander Hörbst

UMIT - Private University for Health Sciences,

Medical Informatics and Technology

Hall in Tirol, Austria

alexander.hoerbst@umit.at

Ghislain B. Kouematchoua Tchuitcheu

IMIA Vice President for HELLNA

kouematchoua@helina-online.org

Dr. Kyung-Hee Cho

President@APAMI.org

Amado Espinosa

IMIA-LAC President

aespinosa@medisist.com.mx

Ramin Moghaddam

MEAHI Supreme Council Chair

Dr_Moghaddam@Meahi.org

IMIA Executive Director:

Elaine Huesing

imia@imia-services.org 